\title{
Dosimetrie eines Blutbestrahlungsgerätes
}

\author{
L. Bogner, P. Härtl, J. Scherer, M. Treutwein, M. Herbst \\ Klinik und Poliklinik für Strahlentherapie und Radioonkologie \\ Universität Regensburg
}

\begin{abstract}
Hintergrund:
Blut und Blutpräparate werden zur Verhinderung der GVHD bei immunsupprimierten Patienten und zur Tumorzellelimination bei der intraoperativen Autotransfusion in der Tumorchirurgie eingesetzt. Für diese Bestrahlungen werden in verstärktem Maße spezielle Gamma-Blutbestrahlungsgeräte verwendet. Der Hersteller liefert für diese Geräte einen Dosisleistungs-Kalibrierwert, meist für den vollständig gefüllten Behälter. Da aber verschiedene Nutzer unterschiedliche Mengen handhaben, ist es nötig, den Einfluß der Füllmenge auf die Absolutdosis in einem Referenzpunkt und die Dosisverteilung im Bestrahlungsvolumen zu untersuchen.
\end{abstract}

Material und Methoden:

An einem mit zwei ${ }^{137} \mathrm{Cs}$-Quellen bestückten Blutbestrahlungsgerät IBL $437 \mathrm{C}$ der Fa. CIS wurde die Dosisleistung in der Mitte des leeren Behälters mit Frickelösungsampullen der Physikalisch-Technischen Bundesanstalt (PTB) gemessen. Mit Hilfe von thermolumineszenzdosimetrischen Vergleichsmessungen (TLD) wurden Anschlußmessungen für verschiedene Befüllungszustände des Bestrahlungsbehälters durchgeführt. So wurde die Dosimetrie eines realistisch befüllten, des komplett gefüllten und des leeren Behälters untersucht. Für die Bestrahlung von Blutbeuteln ist die Kenntnis der Dosisverteilung im bestrahlten Volumen wichtig. Diese Verteilungen konnten im leeren und im mit zwei Blutbeuteln gefüllten Behälter mittels TLD's bestimmt und als Dosis-Volumen-Histogramme (DVH) dargestellt werden. Für den vollständig gefüllten Behälter wurde das MRFricke-Gel-Verfahren zur Messung des DVH's herangezogen.

Ergebnisse:

Die TLD-Messung im Zentrum des vollen Behälters lieferte im Vergleich zum Herstellerzertifikat einen um 4,8\% erhöhten Dosisleistungswert. Aus den Frickelösungsmessungen in Luft in Kombination mit TLDAnschlußmessungen konnten Werte für die Bandbreite der Dosierung bei verschiedenen Füllzuständen gewonnen werden. So ergaben sich für die Grenzwerte leerer und vollständig mit Wasser gefüllter Behälter Über- bzw. Unterdosierungen im Behälterzentrum von 117,5\% bzw. 94\% im Vergleich zu dem mit realistischer Füllung (zwei Beutel) ermittelten Ergebnis. Axiale Dosisverteilungen und DVH's wurden für die 3 Füllzustände ermittelt. Schlußfolgerungen:

Wir empfehlen vor Inbetriebnahme eines Blutbestrahlungsgerätes eine für die jeweilige Befüllungssituation gültige Dosisleistungsmessung zur Bestimmung der Bestrahlungszeiten durchzuführen. Die in dieser Arbeit für eine realistische Füllung und die Grenzwerte leerer bzw. voller Behälter angebenen DVH's erlauben dann eine Abschätzung der Dosisvarianz im bestrahlten Blutvolumen.

\section{Background:}

Blood and blood products are irradiated to avoid the GVHD in immunsuppressed patients and to destroy tumor cells during the intraoperative autotransfusion in tumor surgery. For that purpose more and more dedicated gamma irradiators are used. In most cases the equipment is supplied with a dose calibration factor for a totally filled irradiation cannister. As users handle different blood product volumes, it is necessary to investigate the influence of the irradiated blood volume on the absolute dose in a reference point and the dose distribution in the irradiation volume.

\section{Material and Methods:}

The dose rate in the center of an empty irradiation cannister of a IBL 37C blood irradiator (CIS) was investigated by means of fricke solution dosimeters from the Physikalisch-Technische Bundesanstalt (PTB). Using thermoluminescence dosimetry (TLD) this value could be transferred to a situation with an empty or completely filled respectively with 2 blood samples $(270 \mathrm{ml}$ each) filled cannister. Also essential for the irradiation of blood is the knowledge of the dose distribution in the irradiated volume. The distributions in the empty and the realistic filled cannister were measured by positioning the TLD's on the plexiglas holder in a regular pattern. The case of a completely filled container was investigated by means of the MR fricke gel dosimetry. All distributions are presented as dose-volume-histograms (DVH).

\section{Results:}

The TLD-measurement in the center of the completely filled cannister yielded a $4,8 \%$ higher dose rate value as compared to the suppliers certificate. From the investigations using the fricke solution dosimeters in air combined with TLD-measurements values for the complete bandwidth of different container fillings could be derived. So 
the dose rate in the centre of the cannister in the boundary conditions empty and full cannister as compared to the values for the realistic filling condition ( 2 bags) are 117,5\% and 94\% respectively. Axial dose distributions and DVH's have been determined for the three filling conditions.

Conclusions:

We recommend a dose calibration measurement of a blood irradiator to determine the irradiation times for the chosen filling condition, which is typical for the hospital. The DVH's presented in this work can be used to derive a value for the dose variance within the irradiated blood.

Die Bestrahlung von Blutpräparaten zur Verhinderung der Graft-versus-Host-Erkrankung (GVHD) bei immunsupprimierten Patienten ist seit längerer Zeit eine gängige Methode in der Transfusionsmedizin [1,2,11-13]. Als weitere Anwendung der Blutbestrahlung wird die intraoperative Autotransfusion in der Tumorchirurgie derzeit in klinischen Studien untersucht [6-8]. Infolge vereinzelt aufgetretener Fälle von GVH-Erkrankungen wurde die in der Literatur angebene Dosis von anfänglich 15 Gy bis auf 30 Gy [1,2,10-13] erhöht. Einzelne Autoren empfehlen Dosen von 40 bis zu 50 Gy mit dem Hinweis, daß die eine GVHD auslösende Lymphozytenzahl unbekannt sei $[3,5]$. Bei der Bestrahlung von Blutpräparaten mit Tumorzellkontamination kann mit einer Dosis von 50 Gy eine als ausreichend angesehene Reduktion der Tumorzellen um einen Faktor $10^{-9}$ erreicht werden [6-8]. Es wurde von vielen Gruppen nachgewiesen, daß selbst Dosen von 50 Gy nicht in der Lage sind, Erythrozyten, Thrombozyten, Granulozyten und andere Blutbestandteile wesentlich zu schädigen [6-13].

Derzeit wird die Blutbestrahlung mit Teletherapiegeräten wie ${ }^{60} \mathrm{Co}$ - Geräten und Linearbeschleunigern und in steigendem Maße mit speziellen GammaBlutbestrahlungsgeräten durchgeführt. Da viele Anwender dieser Geräte mit einem hohen Befüllungsgrad des Bestrahlungsbehälters arbeiten, wird das vom Hersteller mitgelieferte Kalibrierzertifikat zur Ermittlung der Dosierung verwendet. Die Hersteller beziehen dabei häufig ihre Kalibrierdosisleistung auf den Mittelpunkt des vollständig mit Wasser gefüllten Behälters. In zwei Arbeiten [9,13] wird auf das Problem der inhomogenen Dosisverteilung über das Bestrahlungsvolumen hingewiesen, auf das manche Anwender mit der Erhöhung der Dosis im Behältermittelpunkt, andere mit einer Einschränkung des Bestrahlungsvolumens reagieren.

In dieser Arbeit wird an einem Gerät der Firma CIS (Typ IBL 437C) untersucht, wie zuverlässig die Angaben des Herstellers zur Absolutdosimetrie und Dosisverteilung im Bestrahlungsbehälter sind. Da in unserer Klinik meist nur 1-2 Blutpräparatebeutel bestrahlt werden, standen die Dosimetrie eines realistischen Befüllungszustandes und die Grenzfälle eines leeren und eines wassergefüllten Behälters im Mittelpunkt des Interesses. Der Beitrag soll damit Abschätzungen über die Situation bei variablen Behälterfüllungen erlauben.

\section{Material und Methoden}

Unsere Klinik verfügt über ein Blutbestrahlungsgerät der Firma Isotopendiagnostik CIS GmbH, Dreieich, vom Typ IBL 437C. Eine schematische Darstellung des Gerätes ist in Abbildung 1 wiedergegeben. Von der maximal möglichen Bestückung von bis zu drei Quellen ist es mit zwei Cäsiumquellen mit je $63 \mathrm{TBq}(1700 \mathrm{Ci})$ ausgerüstet. ${ }^{137} \mathrm{Cs}$ emittiert Gammastrahlung mit einer Energie von $662 \mathrm{keV}$. Die Betastrahlen verlassen die Quellkapselung nicht. Der Bestrahlungsbehälter ist ein Aluminiumzylinder mit einem Volumen von 3,8 1 bei einem inneren Durchmesser von $130 \mathrm{~mm}$ und einer Höhe von $290 \mathrm{~mm}$. Dieser wird in Bestrahlungsposition geschwenkt und dort vor den beiden Quellen mit einer 
Rotationsgeschwindigkeit von 28 Umdrehungen pro Minute gedreht. Der Bestrahlungsvorgang erfolgt nach Eingabe der Bestrahlungszeit vollautomatisch mittels einer elektronischen Steuerung.

Als Referenzpunkt zur Festlegung der Kalibrier-Dosisleistung wurde wie beim Hersteller die Behältermitte gewählt. Das Herstellerzertifikat bezieht sich auf eine Messung der Dosisleistung im wassergefüllten Behälter mit Hilfe der Alanindosimetrie. Um einen präzisen Dosisanschluß an den nationalen Standard herzustellen, bestrahlten wir Ampullen mit Eisensulfatlösung, die wir im Rahmen von Vergleichsmessungen mit chemischen Dosimetern der Physikalisch-Technischen Bundesanstalt (PTB) in Braunschweig erhielten, mit 50 Gy. Diese sogenannte Fricke-Dosimetrie-Methode wird in der Bundesrepublik vielfach als Referenzverfahren zum Dosisanschluß von Therapiebeschleunigern verwendet. Die Ampullen mit einem Durchmesser von $12 \mathrm{~mm}$ und einer Höhe von $32 \mathrm{~mm}$ wurden in der Mitte des leeren Bestrahlungsbehälters in einer Styrodurhalterung positioniert.

Zur Bestimmung der Absolutdosis und der Dosisverteilung in Luft bestrahlten wir Thermolumineszenzdosimeter (TLD) in Plexiglaskapseln, die auf einer Plexiglashalterung befestigt waren (Abbildungen 2a, 2b und 3). In einem weiteren Schritt wurden auf einem Blutbeutel mit ca. $270 \mathrm{ml}$ Inhalt im gleichen Raster TLD's in Kapseln verteilt. Die durchschnittliche Dicke des Beutels betrug 3,5 cm. Die Geometrie der Halterung wurde so gewählt, da in unserer Klinik ausschließlich Erythrozyten- und Thrombozytenkonzentrate zur Applikation in der Hämatologischen Abteilung mit einem typischen Volumen von 200-250 ml und Vollblut mit einem typischen Volumen von 250-300 ml oder 500-600 ml für die intraoperative homologe Transfusion zur Bestrahlung kommen. Mittels der in Abbildung 3 gezeigten abnehmbaren Mittelplatte können diese Präparate - notfalls durch Hinzunahme eines Dummybeutels - einigermaßen reproduzierbar bestrahlt werden. Wir verwendeten individuell kalibrierte TLD100-Rods der Firma STI, Wermelskirchen, deren Reproduzierbarkeit bei einer relativen Standardabweichung von weniger als $1 \%$ lag. Die Auswertung erfolgte im Reader ST5500 der Fa. STI, die Regeneration im Temperofen der Firma PTW, Freiburg. Eine Vergleichsmessung von Eisensulfat und TLD's diente zur Bestimmung des Korrekturfaktors K. Dieser ist ein Produkt aus den Korrekturfaktoren $\mathrm{k}_{\mathrm{q}}$ und $\mathrm{k}_{\text {supra }}$, die sich auf die Abweichung der Strahlenqualität von der Bezugsqualität (6 MV Photonen) und die Supralinearität beziehen. Zur Minimierung des Dosierungsfehlers, der durch die Probeneinfahrzeit und die Rotation mit nicht-ganzzahligen Umdrehungen entsteht, ist eine Dosis von mindestens 5.4 Gy erforderlich. Bei dieser hohen Dosis liefert die Supralinearität einen bedeutenden Beitrag zur Korrektur. Hier konnte auf eine getrennte Bestimmung der Supralinearität verzichtet werden. Die Separation wäre aber prinzipiell möglich [4]. Nach diesem Verfahren ergab sich für K der Wert 0,762. Die Berechnung der Energiedosis in Wasser aus dem TLD-Meßwert erfolgt nach der Gleichung:

$\mathrm{D}[\mathrm{Gy}]=\mathrm{K} \times \mathrm{k}_{\mathrm{ref}} \mathrm{x} \mathrm{N} \times \mathrm{M}$

Dabei stellt $\mathrm{k}_{\mathrm{ref}}$ den Bezug zu einer am Meßtag durchgeführten Referenzbestrahlung her, $\mathrm{N}$ den Kalibrierfaktor bezüglich der Bezugsstrahlenqualität und M die Meßanzeige dar.

Neben der Absolutdosis zur Festlegung der Bestrahlungszeit ist die Kenntnis der Dosisverteilung im Blutvolumen von entscheidender Bedeutung. Der Hersteller gibt eine Verteilung an, die für ein Gerät mit 3 Quellen in einem Näherungsverfahren berechnet wurde. Zur Untersuchung der Relativdosis, die auf die Dosis im Referenzpunkt bezogen wird, haben wir verschiedene Verfahren verwendet. Dosisverteilungen im leeren Behälter und bei Befüllung mit zwei Blutbeuteln wurden mit TLD's in der in den Abbildungen $2 \mathrm{a}, 2 \mathrm{~b}$ und 3 gezeigten Vorrichtung gemessen. Außerdem überprüften wir die vom Hersteller angebene 
Dosisverteilung in einem wassergefüllten Bestrahlungsbehälter mit der MR-Fricke-GelDosimetrie. Darunter versteht man eine Methode, bei der durch Gelierung einer Eisensulfatlösung mittels Gelatine die Eisenionen räumlich fixiert werden. Die bei der Deposition der Strahlungsenergie lokal auftretende Oxidation des Eisenions bewirkt eine dosisproportionale Änderung der Magnetresonanz-Relaxationsraten. Die Auswertung in einem MR-Tomographen liefert einen Volumensatz von T2-Relaxationszeiten, der in eine räumlich hochaufgelöste Dosisverteilung umgerechnet werden kann. Vorteilhaft für den Einsatz im Blutbestrahlungsgerät ist die hohe Linearität der Methode bis zu einer Dosis von 40 Gy [14]. Die Dosisverteilungen wurden zur übersichtlichen Darstellung in Form von integralen Dosis-Volumen-Histogrammen (DVH), die dem Integral über die Häufigkeitsverteilung der in der Probe vorkommenden Dosen entspricht, angegeben. Für die Auswertungen der MR-Fricke-Gel- und TLD-Luft-Messungen wurden wegen der Rotationssymmetrie nur die Ergebnisse der mittleren Schicht (Abbildung 2 b) verwendet. Die Umrechnung des resultierenden Dosis-Flächen-Histogramms in ein DVH erfolgte mittels Aufteilung des Bestrahlungsvolumens in Voll- und Hohlzylinder. Bei der Messung mit Blutbeutel wurde dieser näherungsweise als Quader betrachtet und für die DVH-Berechnung in Teilvolumina, die je 1 TLD enthalten, zerlegt.

\section{Ergebnisse}

Zur Überprüfung der vom Hersteller im Kalibrationszertifikat angebenen Dosisleistung in der Mitte des voll mit Wasser gefüllten Bestrahlungsbehälters, die mit der Alanindosimetrie, bestimmt wurde, haben wir unter den gleichen Bedingungen eine TLD-Messung durchgeführt. Die Alanindosimetrie wurde vom „Laboratoire de Mesure des Rayonnements Ionisants“, einem französischen Sekundär-Standardlabor, durchgeführt.

Um die Bandbreite von möglichen Dosierungsfehlern abzuschätzen, die sich durch eine von den Kalibrierbedingungen abweichende Befüllung des Behälters ergeben, haben wir zwei extreme Grenzfälle untersucht. Der eine Grenzfall bei vollständiger Füllung mit Wasser wurde bereits vorgestellt. Der andere Grenzfall liegt bei vollständig leerem Behälter vor. Wir haben in den Jahren 1993 und 1994 Messungen mit Eisensulfatlösungen der PTB in der Mitte des leeren Behälters durchgeführt. Aus dem Mittelwert beider Messungen wurde eine LuftDosisleistung bestimmt, die als Ausgangswert für die Bestimmung unseres Kalibrierfaktors für die Routinebestrahlung diente. Nach Angaben der PTB ist die Methode für ${ }^{60} \mathrm{Co}$ - und Bremsstrahlung von $1-50 \mathrm{MeV}$ geeignet und energieunabhängig. Bei der Anwendung auf 662 $\mathrm{KeV}$-Gammastrahlung gehen wir davon aus, daß hier keine wesentlichen Abweichungen auftreten. Weiterhin wird angenommen, daß sich bei der geringen Energie bereits innerhalb der Glasumkapselung der Frickelösung ein Sekundärelektronengleichgewicht einstellt und deshalb keine weitere Korrektion für die Abweichung von der Bezugsbedingung - Kalibration mit ${ }^{60} \mathrm{Co}$ in Wasser - nötig ist. Der direkte Weg eines Anschlusses des Blutbestrahlungsgerätes mit Frickedosimetern in Wasser wurde aus pragmatischen Gründen wegen der Rotation des Bestrahlungsbehälters vermieden.

Da wir im allgemeinen einen oder zwei Konzentrat- oder Blutbeutel mit einem Volumen von je etwa 200-300 ml gleichzeitig bestrahlen, wobei bei der Bestrahlung mit einem Beutel dann ein wassergefüllter Beutel den zweiten Einschub auffüllt, ermittelten wir für diese Geometrie die Kalibrations-Dosisleistung. Dieses Ergebnis kann auch für die Bestrahlung von einem Vollblutbeutel mit einem Volumen von etwa 500-600 ml verwendet werden, wenn der in der Abbildung 3 gezeigte Mittelsteg entfernt wird. Neben der Kalibrationsvorrichtung sind die beiden für die Routinebestrahlung verwendeten Styrodureinsätze abgebildet. Es wurde eine TLD-Vergleichsmessung im Zentrum des leeren und des mit zwei Beuteln mit je $270 \mathrm{ml}$ gefüllten Behälters durchgeführt. Mit Hilfe der Eisensulfat-Luft-Dosisleistung und des aus der 
TLD-Messung bei Befüllung mit Blutbeuteln gewonnenen Korrekturfaktors erhält man die in der Praxis verwendete Kalibrier-Dosisleistung $\mathrm{DL}_{(\text {Beutel })}$.

$\mathrm{DL}_{(\text {Beutel })}=\mathrm{DL}_{\mathrm{FeSO} 4(\mathrm{Luft})} \times\left(\mathrm{D}_{\mathrm{TLD}(\text { Beutel })} / \mathrm{D}_{\mathrm{TLD}(\mathrm{Luft})}\right)$

Die Ergebnisse der Absolutdosimetrie, die sich bei der Verwendung von grenzwertigen Behälterfüllungen ergeben, sind mit den prozentualen Abweichungen in Tabelle 1 wiedergegeben.

DVH's stellen die Verteilung der Dosis über das bestrahlte Volumen statistisch dar. Zur Untersuchung der Grenzfälle Wasserfüllung und leerer Bestrahlungsbehälter wurde zum einen mit der MR-Fricke-Gel-Dosimetrie die Dosisverteilung im vollständig mit Gel gefülltem Behälter bestimmt. Fricke-Gel kann in Bezug auf Zusammensetzung (95\% Wasser) und Dichte $\left(1,05 \mathrm{~g} / \mathrm{cm}^{3}\right)$ in guter Näherung für Wasser verwendet werden. Zum anderen wurden Messungen mit TLD's im leeren und im mit zwei Beuteln gefüllten Behälter durchgeführt. Abbildung 4 zeigt die axiale Verteilung der Dosis. In den Abbildungen $5 \mathrm{a}$ und $5 \mathrm{~b}$ sind die vom Hersteller für drei Quellen berechnete und die mit der MR-Fricke-Gel-Methode für zwei Quellen gemessene Isodosenverteilung in einem Schnitt durch die Rotationsachse dargestellt. Das zugehörige DVH ist in Abbildung 6 a enthalten. In Abbildung 6 b sind die DVH's für die Grenzfälle Luft- und Wasserfüllung für das nach Abbildung 2 a und $2 \mathrm{~b}$ durch die TLDPositionen definierte Meß-Teilvolumen angegeben. Abbildung 6 c zeigt ein DVH für die praxisnahe Befüllung mit zwei Beuteln. Die Dosis ist in allen Fällen auf die Werte in der Behältermitte normiert. Die Standardabweichung der TLD-Dosis am Blutbeutel, die sich aus der TLD-Reproduzierbarkeit und der Positionierungenauigkeit des Beutels ergibt, liegt bei $1,6 \%$.

\section{Diskussion und Schlußfolgerung}

Eine Überprüfung der Herstellerangaben zur Dosisleistung im Zentrum des Bestrahlungsbehälters ergab einen Unterschied von 4,8\%. Der Hersteller gibt mit der mitgelieferten berechneten Dosisverteilung im Bestrahlungsvolumen bereits einen Hinweis auf mögliche Dosisvarianzen im Blutvolumen. Für Anwender mit hohem Bestrahlungsaufkommen, d.h. bei relativ voll gefülltem Behälter, kann der durch eigene Dosimetrie überprüfte Zertifikatswert verwendet werden, wenn man sich bewußt ist, daß Blutanteile Dosen zwischen 85\% und 125\% dieses Wertes erhalten können. Eine genauere Analyse der Dosisverteilungsstatistik stellt das DVH dar, das die Volumenanteile mit den zugehörigen Dosen angibt. Das Bestrahlungsaufkommen in unserer Klinik ist relativ gering, so daß maximal zwei Blutbeutel gleichzeitig zur Bestrahlung kommen. Deswegen und auch um schwankende Befüllungszustände abschätzen zu können, haben wir für die Grenzwerte eines leeren und eines vollen Behälters die Dosisleistung im Zentrum genauer untersucht. Es ergaben sich Unterschiede von 117,5\% bzw. 94\%, bezogen auf den realistischen Fall mit zwei Beuteln (je $270 \mathrm{ml}$ ). Die Darstellung der Dosisverteilungen mit DVH für diese drei Fälle gibt eine Möglichkeit an die Hand, die Volumenanteile mit Unterdosierungen - bezogen auf die auf $100 \%$ normierte Mittelpunktsdosis - abzuschätzen. Für die realistische Befüllung besagt das DVH in Abbildung 6 c, daß 100\% des Blutvolumens mehr als 95\% (bis zu 125\%) der Kalibrierdosis erhält. Diese Werte beziehen sich auf die Geometrie, die für die Routinebestrahlung mit dem in Abbildung 3 gezeigten Styrodureinsatz gegeben ist. Aus dem DVH des vollen Behälters (Abbildung 6 a), das sich auf das gesamte Behältervolumen bezieht, ergibt sich, daß dieses Volumen mehr als 85\% (bis mehr als 125\%) der Kalibrierdosis erhält. Beschränkt man sich auf das in Abbildung 2 a und $2 \mathrm{~b}$ durch die TLD markierte 
Teilvolumen, so erhielte dieses bei leerem bzw. bei vollem Behälter mehr als 95\% bzw. 100\% ( bis zu 120\% bzw. über 125\%) der Kalibrierdosis (Abbildung 6b).

Aufgrund dieser Erfahrungen empfehlen wir, durch entsprechende Messungen die Absolutdosis für eine vorgewählte Bestrahlungsgeometrie bei festem Befüllungsvolumen zu bestimmen und aus den DVH in den Abbildungen $6 \mathrm{a}, 6 \mathrm{~b}$ und $6 \mathrm{c}$ eine Abschätzung der darin vorhandenen Dosisverteilung durchzuführen. Bei großen Blutmengen empfiehlt es sich, aus dem DVH (Abbildung 6 a) einen Dosiserhöhungsfaktor zu bestimmen, der sicherstellt, daß ein vorwählbares Teilvolumen eine bestimmte Mindestdosis erhält. Bei kleinen Mengen genügt es, das Bestrahlungsvolumen mit Vorrichtungen auf die achsennahen Areale zu verkleinern.

Die von Hansen et al. [6-8] durchgeführten Bestrahlungen bei der intraoperativen Autotransfusion wurden an unserem Blutbestrahlungsgerät unter Zuhilfenahme der hier vorgestellten Dosimetrie durchgeführt.

Literatur

1. Adamietz IA, Müller R., Seidel P. et al. Die prophylaktische Bestrahlung von Blutkomponenten mit herkömmlichen Strahlentherapiegeräten, Strahlenther. Onkol. 1989;165:855-9.

2. Button LN, DeWolf WC, Newburger PE, et al. The effects of irradiation on blood components, Transfusion 1981;21: 419-426.

3. Drobyski W, Thibodeau S, Truitt RL, et al. Third party-mediated graft reaction and graft-versus-host disease after T-cell-depleted bone marrow transplantation, as demonstrated by hypervariable DNA probes and HLADR polymorphism, Blood 1989;74:2285-94 .

4. Feist, H. Einfluß des Regenerier- und Auswerteverfahrens auf das supralineare Verhalten von LiFThermolumineszenzdosimetern. Strahlenther Onkol 1988;164:223-7.

5. Goldmann SF, Ebell W, Blütters-Sawatzki R, et al. Graft-versus-Host-Reaktion (GvHR) nach perinataler materno-fetaler Transfusion bzw. nach Substitution mit Blutzellen: Der diagnostische Wert der HLATestung. Beitr Infusionsther Klin Ernähr 1987;18:321-5

6. Hansen E, Hofstädter F, Taeger K et al. Autologe Transfusion bei Tumoroperationen, Infusionsther. Transfusionmed 1994;21:337-47.

7. Hansen E, Knuechel R, Kutz N et al. Elimination von Tumorzellen in Wundblut durch Bestrahlung. Hämatologie Bd.4 München: Sympomed 1995: 22-7.

8. Hansen E, Taeger K. Intraoperative Autotransfusion mit Blutbestrahlung bei Tumoroperationen: Grundlagen und Praxis, Anästhesiol Intensivmed 1996;37:306-12.

9. Kaulich TW, Kübler MJH, Nüsslin F. Untersuchungen zur Dosishomogenität bei Bestrahlung von Blutkomponenten, Tagung Medizinische Physik, Basel, 1992:266-7.

10. Leitman SF. Dose, dosimetry, and quality improvement of irradiated blood components. Transfusion $1993 ; 33: 447-9$

11. Leitman SF, Holland PV: Irradiation of blood products, Transfusion 1985;25:293-300

12. Linden JV, Pisciotto PT. Transfusion-associated graft-versus-host disease and blood irradiation, Transfus Med Rev 1992;6:116-23

13. Masterson ME, Febo R. Pretransfusion blood irradiation: Clinical rationale and dosimetric considerations, Med Phys 1992;19:649-57

14. Scherer J, Bogner L, Herbst M et al. Verifikation optimierter 3D-Dosisverteilungen mittels MR-Fricke-Gel, Strahlenther Onkol 1997;173:36-42

Korrespondenzanschrift:

Dr. Ludwig Bogner

Klinik und Poliklinik für Strahlentherapie und Radioonkologie

Universitätsklinikum

Franz-Josef-Strauß-Allee 11

D-93042 Regensburg 


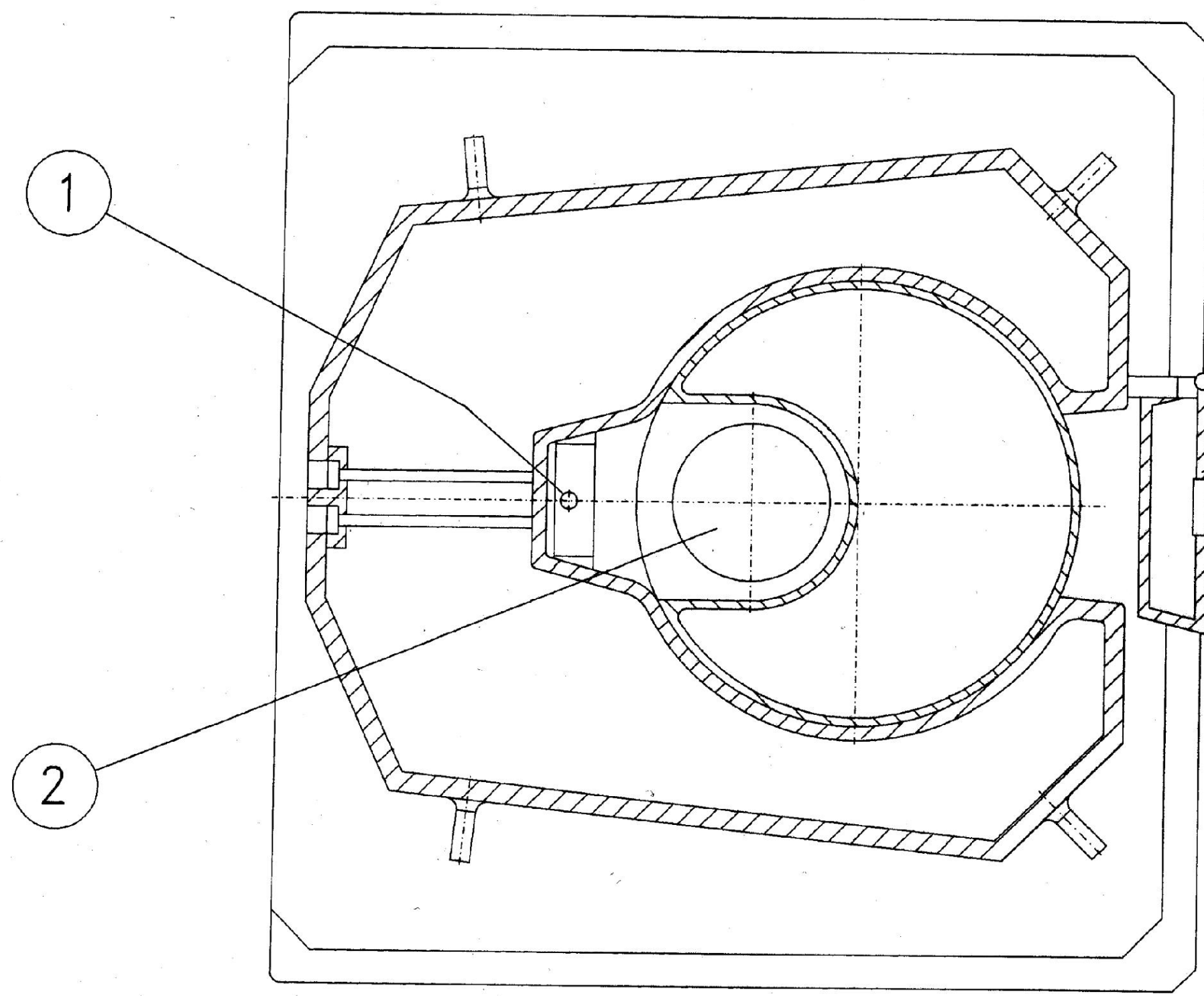

Abbildung 1. Schematische Darstellung des Blutbestrahlungsgerätes IBL 437C der Firma CIS (1: Quellkapsel; 2: Bestrahlungsbehälter in Bestrahlungsposition; 3: Beladungstür).

Figure 1. Schematic drawing of the irradiator IBL 437C (CIS Diagnostik) $(1=$ radiation source; 2 = canister in radiation position; 3 = door). 

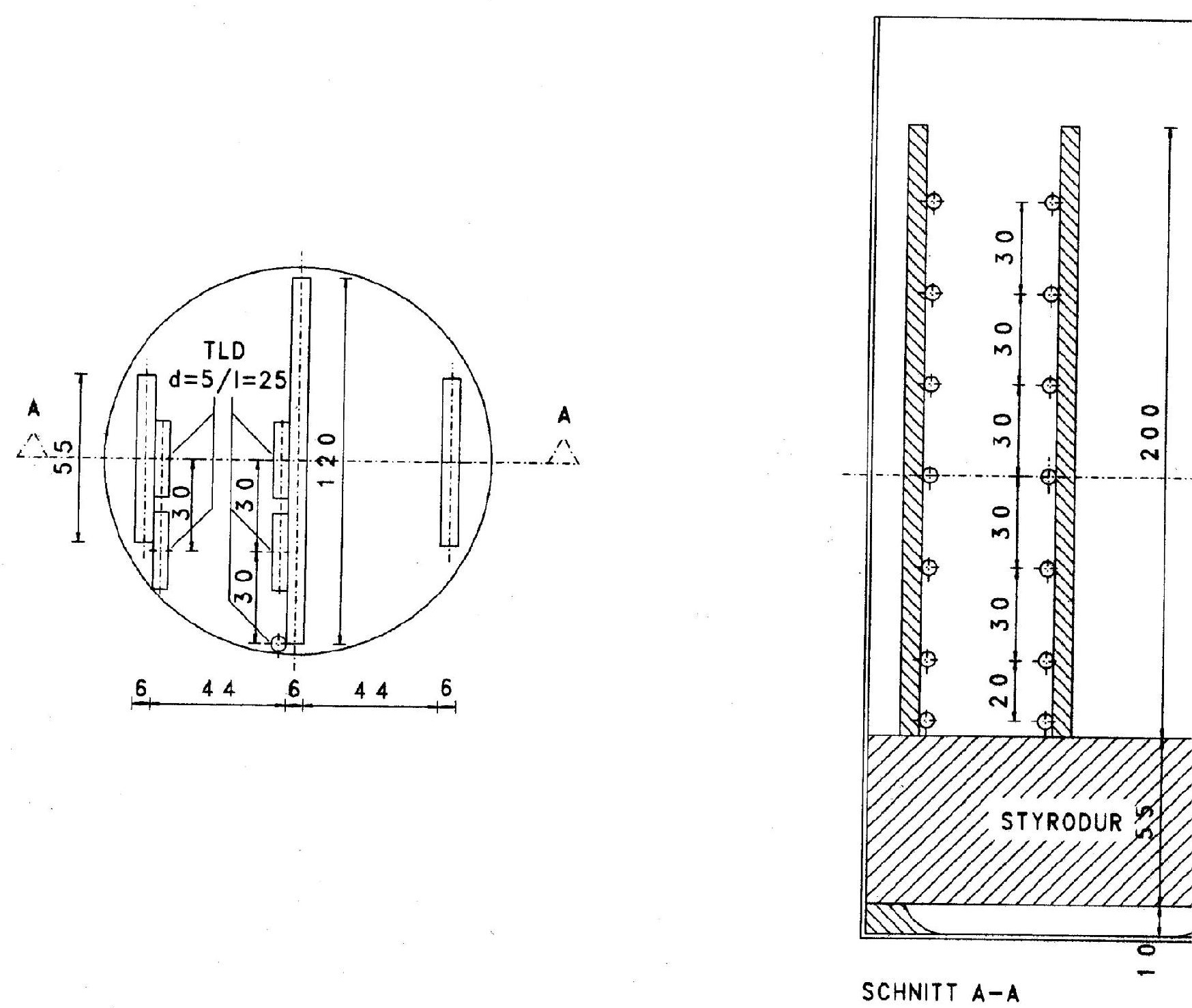

Abbildungen 2a und 2b. Draufsicht (a) und Längsschnitt (b) des für die Messungen verwendeten Plexiglaseinsatzes. Eingetragen sind die Meßpositionen der TLD-Kapseln.

Figures $2 \mathrm{a}$ and $2 \mathrm{~b}$. Top view (a) and cross section (b) of the canister with perspex holder for the TLDs. The position of the TLD capsules are marked. 


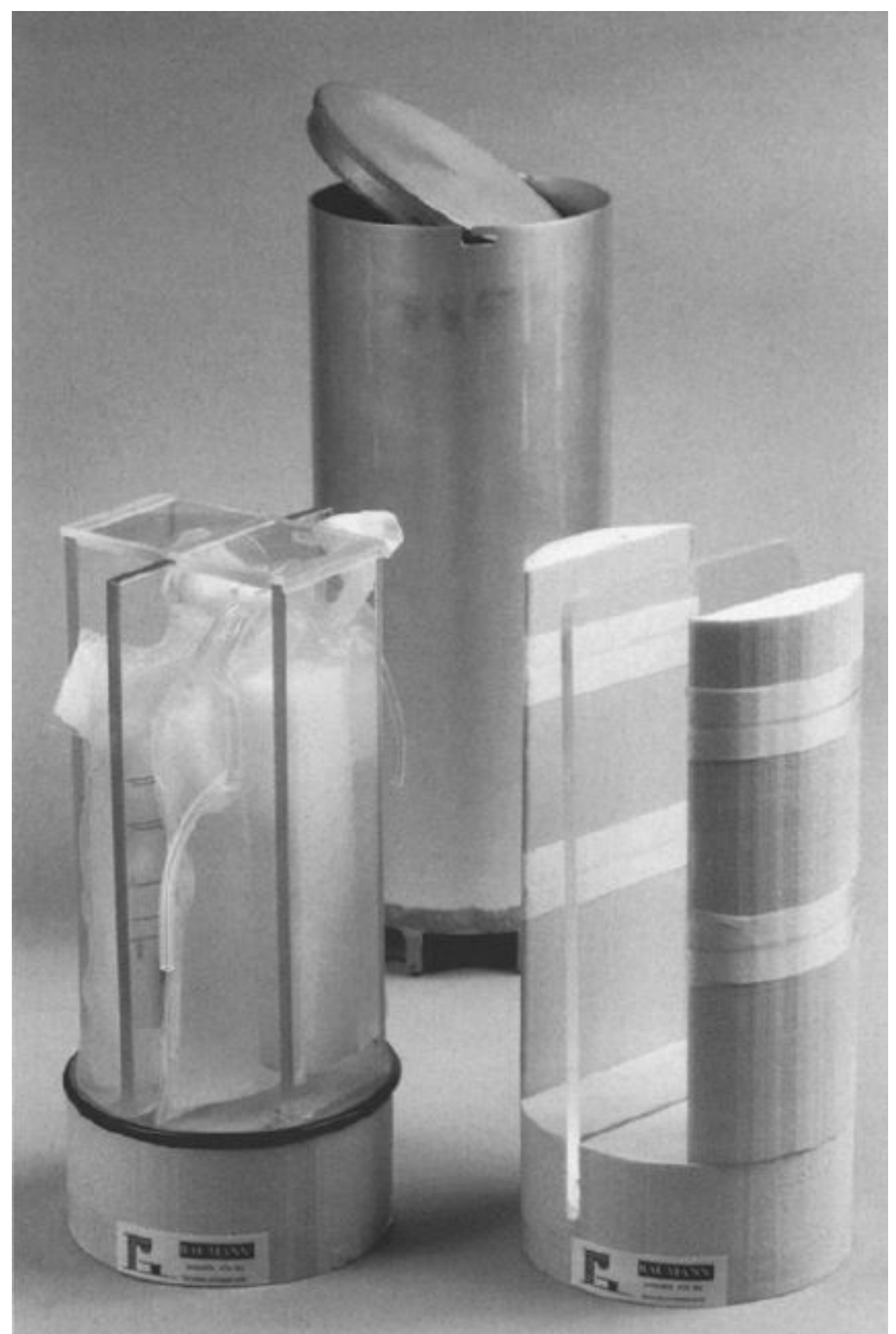

Abbildung 3. Ansicht des Bestrahlungsbehälters und des mit zwei Blutbeuteln gefüllten Meßeinsatzes sowie der Styrodurvorrichtung für Routinebestrahlung.

Figure 3. Left: the holder filled with 2 blood bags. Right: the holder of styrofoam and perspex for the daily work. Background: canister. 


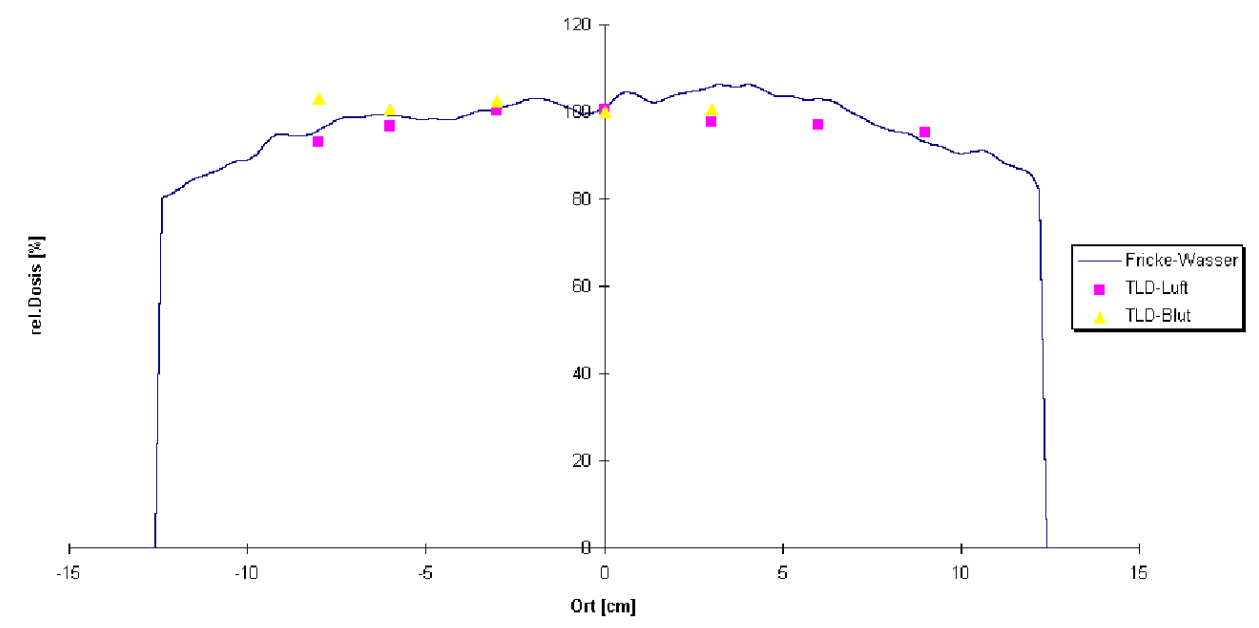

Abbildung 4. Axiale Dosisverteilung im leeren, vollen und mit zwei Blutbeuteln (je $270 \mathrm{ml}$ ) gefüllten Bestrahlungsbehälter.

Figure 4. Axial dose distribution in the empty (black squares), water filled (closed line) and with 2 blood bags filled canister $270 \mathrm{ml}$ both (white triangles).
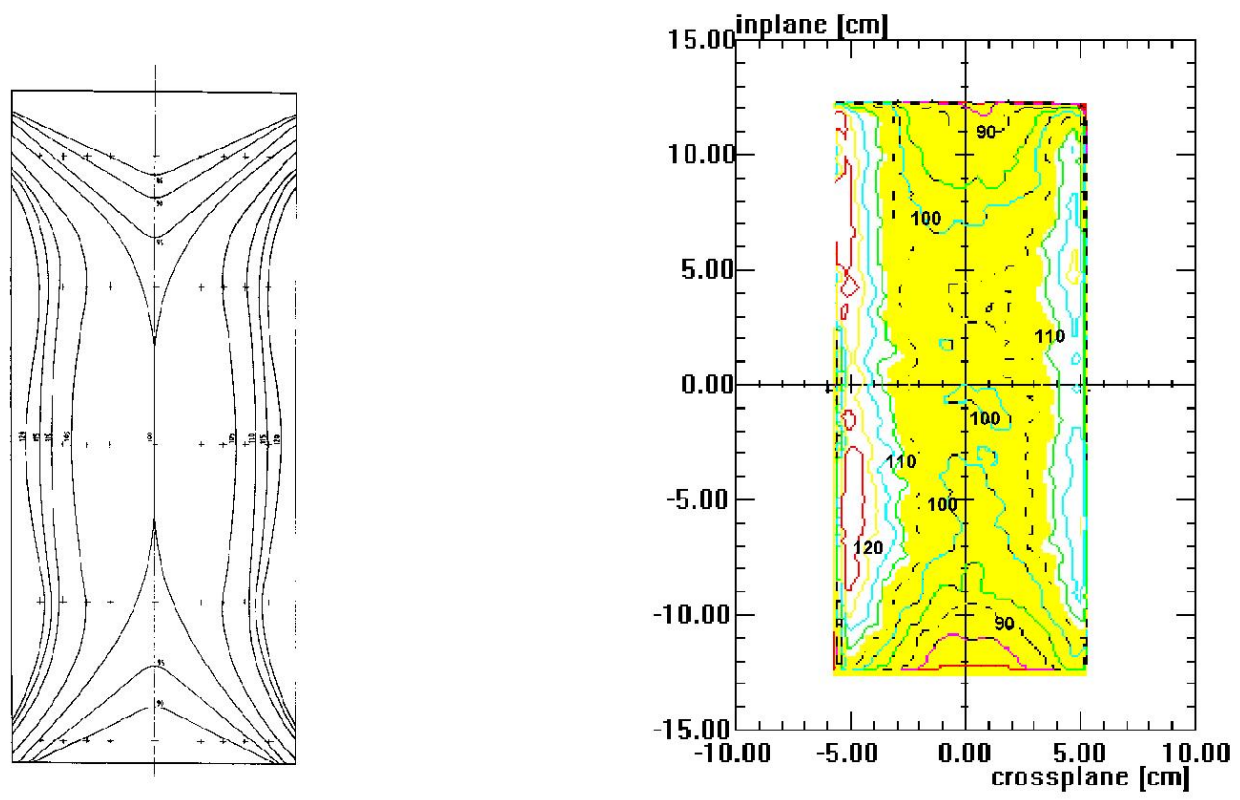

Abbildungen 5a und 5b. Vom Hersteller für drei Quellen berechnete (a) und mit MRFricke-Gel-Dosimetrie für zwei Quellen gemessene (b) Dosisverteilung in einem Schnitt durch den Bestrahlungsbehälter.

Figures $5 \mathrm{a}$ and $5 \mathrm{~b}$. Calculated dose distribution of the manufacturer for 3 sources (a) and measured by Fricke gel dosimetry for 2 sources (b) in a cross section. 

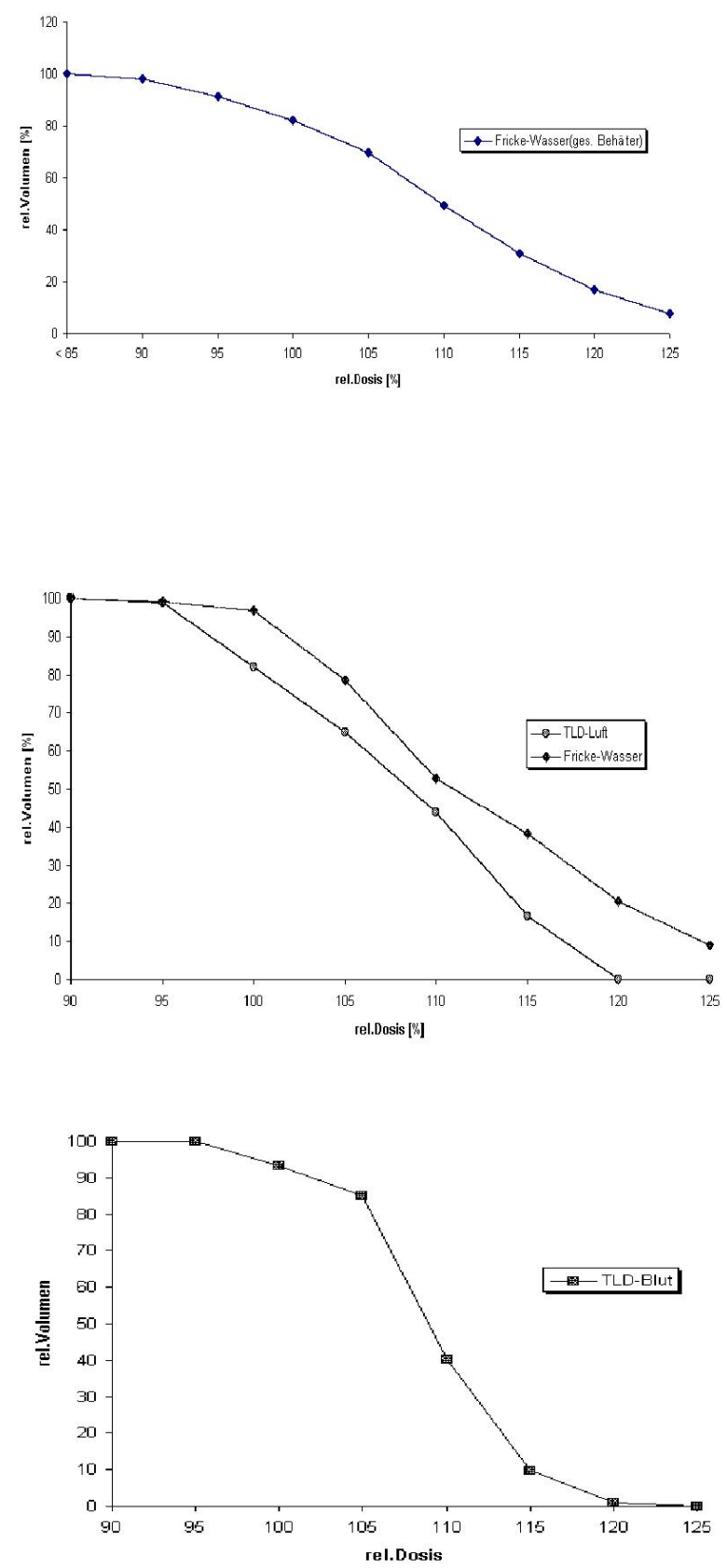

Abbildungen 6a bis 6c. Dosisvolumenhistogramme für den vollen Behälter (a), die Grenzfälle des vollen und leeren Behälters (b) sowie der mit zwei Blutbeuteln (je $270 \mathrm{ml}$ ) gefüllten Vorrichtung (c). Die relativen Volumina sind bei (a) auf das Gesamtvolumen, bei (b) auf das eingeschränkte Meßvolumen und bei (c) auf das Blutvolumen bezogen.

Figures $6 \mathrm{a}$ to $6 \mathrm{c}$. Dose-volume histograms for the filled canister referring to the total volume (a), the filled and the empty canister referring to the shrinked measuring volume (b) and containing 2 blood bags ( $270 \mathrm{ml}$ both) referring to the blood volume (c). 


\begin{tabular}{|c|c|c|c|}
\hline Meßmethode & Behälterfüllung & Dosisleistung (Gy/ min) & $\begin{array}{c}\text { Relative } \\
\text { Dosisleistung /\% }\end{array}$ \\
\hline Alanin (Hersteller) & Wasser & $6,42 \pm 5,5 \%$ & 90 \\
\hline TLD & Wasser & $6,73 \pm 5,0 \%$ & 94 \\
\hline Eisensulfat 1993 & Luft & $8,34 \pm 2,1 \%$ & 117 \\
\hline Eisensulfat 1994 & Luft & $8,45 \pm 2,1 \%$ & 118 \\
\hline TLD & $\begin{array}{c}\text { 2 Blutbeutel }(\mathrm{je} \\
270 \mathrm{ml})\end{array}$ & $7,15 \pm 5,0 \%$ & $\mathbf{1 0 0}$ \\
\hline
\end{tabular}

Tabelle 1. Absolutdosimetrie: Übersicht über die bei verschiedenen Füllungszuständen im Behältermittelpunkt gemessenen Dosisleistungen (Bezugszeitpunkt ist das Zertifikatsdatum). Der Herstellerwert ist nach Zertifikat mit dem absoluten Fehler angegeben, die übrigen Fehlergrenzen bezeichnen das 95\%-Vertrauensniveau.

Table 1. Absolute dosimetry: overview of the dose rate in the middle of the canister for 3 different filling conditions (calculated for the date of the source certificate). The value of the manufacturer is given with absolute errors, the other error limits mark the $95 \%$ confidence interval. 